\title{
Integration of Domain Ontologies in the Repository of Website Evaluation Methods
}

\author{
Paweł \\ Ziemba $^{1}$ \\ Jarosław \\ Jankowski ${ }^{2,3}$ \\ Jarosław
Wątróbski $^{2}$ \\ Waldemar \\ Jarosław \\ Wolski $^{4}$ \\ Becker $^{1}$
${ }^{1}$ The Jacob of Paradyż University of Applied Sciences in Gorzów Wielkopolski, Chopina 52, 66-400 Gorzów Wielkopolski, Poland
Email: \{pziemba, jbecker\}@pwsz.pl
${ }^{2}$ West Pomeranian University of Technology in Szczecin, Żołnierska 49, 71-210 Szczecin, Poland
Email: \{jjankowski, jwatrobski\}@wi.zut.edu.pl
${ }^{3}$ Department of Computational Intelligence, Wrocław University of Technology, Wybrzeże Wyspiańskiego 27 , 50-370 Wrocław, Poland
${ }^{4}$ University of Szczecin, Mickiewicza 64, 71-101 Szczecin, Poland
Email: wwolski@uoo.univ.szczecin.pl

\begin{abstract}
Many methods can be used for the evaluation of website quality. While they can be used for different purposes and require different assessment approaches it is not easy to select proper method adequate to the needs. Presented research is focused on building a repository of knowledge about the methods for the assessing the quality of website. The repository in the form of ontologies covers various methods of quality assessment and makes possible their proper selection. Proposed approach was verified with main methods and the resulting ontology can act as a repository of domain knowledge.
\end{abstract}

\section{INTRODUCTION}

$\mathrm{I}^{\mathrm{n}}$ the sectors related to e-commerce and online advertising, the number of users visiting corporate websites, blogs, portals and social platforms directly determines revenue. More users increase the potential of advertising, which has a direct impact on the number of transactions, the amount of revenue, and the ability to engage new customers [1]. In the United States, recent revenues from online advertising amounted to 42.78 billion dollars [2], while in Europe, it amounted to 27.3 billion euros in the advertising sector [3] and 363,1 billion euros in e-commerce [4]. It is worth noting that for businesses using a website to generate transactions, the website's quality can have a major impact on sales [5]. Poor quality of service and user experience can cause existing Internet customers [5], potential sales, and repeated visits to be lost [6]. Therefore, in order to maximize profits from e-commerce or online advertising, website owners should only offer the highest quality experience and services.

The quality of a website can be understood as the qualification of how well it meets the needs of users [7]. It should be noted that quality is defined by a model composed of characteristics and features/criteria describing its various components [8]. In the literature, there are many methods used to assess the quality of Internet websites, with the most formalized of those including: eQual [9], Ahn [10] SiteQual [11], Web Portal Site Quality [12] and Website Evaluation Questionnaire [13]. They have been widely used in both academic work [14] and business practice [15]. By examining the different methods was observed similarity between them. Characteristics and criteria for quality assessment based on a Likert scale were used and for the getting knowledge about quality of services surveys were explored. The reason for these similarities is that different methods are often based on the same scientific theories and source references.

Analysis of the literature and areas of practical use of methods and models for the assessment of website quality indicates a gap in the area of knowledge repository construction. Namely, there is no repository of knowledge covering some important methods for assessing the website quality. The possible construction of such a repository in the form of ontology allows formal specification and analysis of the various methods of assessment and factors affecting the quality of website [24], as well as consequent sharing and reusing that domain knowledge [17]. The ontological form provides access to the knowledge gained from individual assessment methods and enables the processing of this knowledge. It is also important to enable the integration of heterogeneous data [18] from a variety of assessment methods. As a result, it will also be possible to assess websites through a variety of methods defined in the ontology and compare individual results of that assessment in a single terminology and a reference plane. In this approach, the problem of building such a repository is divided into two subproblems. They successively concern (1) the construction of the source ontologies, reflecting the 
specific methods for quality assessment; (2) the integration of source ontologies in the target ontology, which is a complete repository. This approach is justified by the abovementioned similarities between the different methods, so that individual source ontologies are close to each other in terms of structure and concepts. Proposed approach of building a repository of knowledge is graphically represented in Figure 1 .

This article presents guidelines on how to design an ontology reflecting the various quality assessment methods and their integration in the repository of knowledge. Then, using these assumptions new algorithm was proposed based on the integration of the five methods in the repository. The article concludes with a presentation of research findings and possible future directions for work.

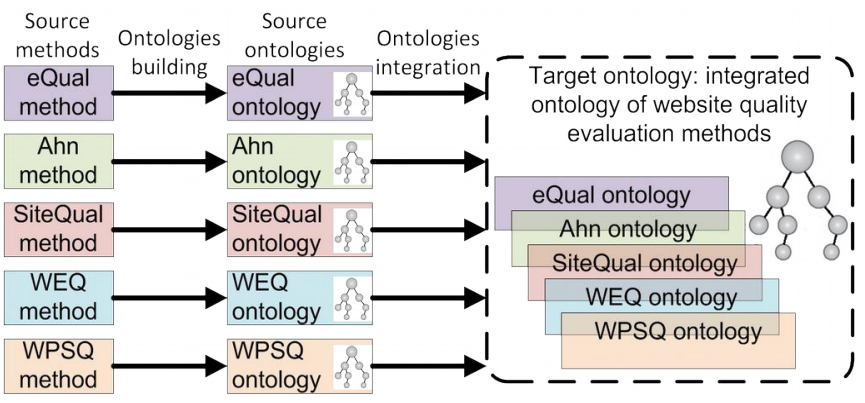

Fig 1. Proposed approach to building repository of knowledge by integration of ontologies

\section{LITERATURE REVIEW}

The possibility of constructing a repository of knowledge on the methods and models of assessing the quality of Internet services in the form of ontologies confirms its definition, as the term "ontology" in computer science is defined as the "formal specification of conceptualization" [19]. Essentially, it allows concepts and domain knowledge to be captured. A similar definition states that ontology is treated as a form of data structure and a tool for data representation, allowing knowledge to be shared and reused in artificial intelligence systems that use a common vocabulary [20]. Therefore, ontology seems to be a natural form of representation for the repository of knowledge concerning methods of quality assessment. This is due to the fact that the use of ontologies will create conceptual models that explain the structure of the different methods of evaluation criteria. The use of ontologies will also be shared, making it possible to repeat the use of such structures and facilitate better management.

The possibility of using ontologies as a repository of knowledge is confirmed by various studies where among others a biomedical knowledge base was created using ontologies [21]. Ontologies are also implemented in the knowledge bases of various systems, e.g. an expert system for the study of company financial ratios [22] and a decision support system for the construction of railway infrastructure
[23]. Analysis of the literature shows that ontologies are also used in the systems and methods of quality assessment. For example, [24] presents an ontology quality that formalizes the knowledge necessary to evaluate the quality of egovernment. In contrast, [25] proposes the use of ontologies in the quality assessment of tourism services websites. In this case, the ontology serves as a repository of knowledge on issues related to the tourism sector. However, analysis of the literature showed that there is no repository of knowledge covering some important methods for assessing the quality of website. Meanwhile, the reasons discussed above and the use of ontologies both demonstrate that the construction of a repository of knowledge about the methods of assessing website quality based on ontologies is justified.

The ontology construction methodology is frequently used, although it differs in the degree of formalization, destination and detail [26]-[30]. The most formal and detailed methodology includes Methontology [31] and NeOn [32]. Methontology proposed an "ontology life cycle" based on the so-called "evolving prototypes" [29]. This approach assumes that the individual steps of this methodology, namely I specification, II conceptualization, III formalization, IV implementation, and V maintenance, are performed continuously, and each cycle is replaced by a new version of the ontology. The NeOn methodology is focused on developing an ontology network instead of a single ontology. An ontology network is understood here as a set of related ontologies through a variety of compounds, e.g. mapping and versioning [26]. Methontology defines the process of conceptualization in detail, while NeOn largely formalizes the problem of ontology specification.

Concerning the integration of ontologies, it should be noted that the concept of integration is not clearly defined in different works. Reference [33] distinguishes three types of ontology integration: integration of ontologies into applications, integration and reusing, and integration by merging. Integration and reusing is defined as the construction of a new ontology by using existing ontologies. Merging is the unification of multiple ontologies from a given field into a new ontology. Merged ontologies may vary in appearance and function, including taxonomy of concepts, method of implementation, etc. Conflicts arising from these differences should be resolved when merging ontologies [33]. Reference [34] stated that ontology created by such a merger should capture all the knowledge contained in the original ontologies. Two approaches may be used: a new ontology can be created that reflects the source ontologies, or what is known as bridge ontology can be created. Bridge ontology contains the original ontologies and the relationship between them. In [35] integrating and merging operations are defined as equal. They include the creation of a new ontology from two or more existing ontologies with overlapping parts. In addition, reference [34] defines the terms being similar to integration, i.e. alignment and mapping. Alignment is a preliminary process by which it is 
possible to integrate ontology in a widely understandable way. It is a process of discovering similarities between ontologies. These similarities may occur between the concepts, their instances, or a similarity in the ontology structure. Mapping, on the other hand, refers to representing the relationships that exist between ontologies. As result a specification of the semantics of one ontology coverage by another is obtained which can be represented as a map describing the mutual relations between the elements of mapped ontology). Relationships between ontologies are stored separately from the ontology and are not part of them. Therefore, the mapping does not change in any of the ontology. Mapping allows to get a result somewhat similar to bridge ontology. However, in bridge ontology, as opposed to mapping, source ontologies and connections between them are stored in one entity.

\section{Construction OF THE SOURCE ONTOlOGies}

In order to successfully complete integration, it is necessary to construct an appropriate source ontologies, reflecting the various website quality assessment methods. Consequently, the design of the source ontology is affected by the structure of the quality models included in the assessment methods and aspects relateted to their future integration. As indicated in Paragraph 1, each of the methods of evaluation is based on the quality model composed of characteristics and features/criteria describing its various components. Each of the source ontologies should reflect the quality model, which can be represented by a general structure, as illustrated in Figure 2.

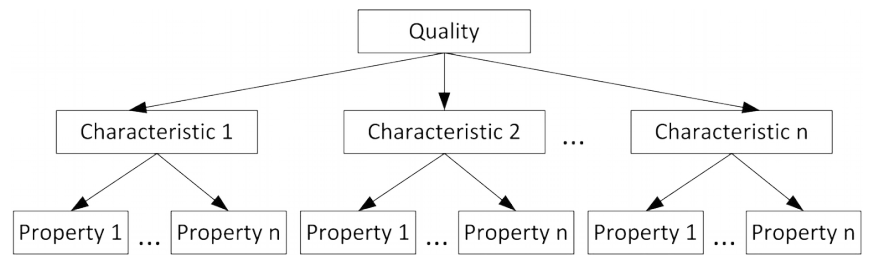

Fig 2. General quality model
With regard to the structure of the ontology generated by integration, it is assumed that the target (integrated) ontology should contain a unified model, including all the characteristics and evaluation criteria, and simultaneously contain therein quality models derived from different methods. The purpose of storing individual quality assessment models (source ontologies) in the target ontology is to determine which method and characteristics derives a given criterion. Ability to determine the source method of a given criterion allows the integration of heterogeneous data from a variety of assessment methods into knowledge base. The inclusion in the ontology of a unified model allows to use it as a knowledge repository on evaluation criteria. Therefore, the ancestor of each criterion should be a model from which the criterion is derived together with the characteristics. In addition, if certain criteria are found in many different models of assessment contained in the ontology, then it will take more than two direct ancestors. This problem is illustrated in Figure 3.

Figure 3 shows part of the quality characteristics contained in Ahn and eQual methods. Moreover Figure 3 also includes a hypothetical model, which is a result of the unification of the ontologies of these methods. Three further examples of criteria are included, of which "availability" is derived from the Ahn method, "ease_of_learn_to_operate" comes from the eQual method, and "ease_of_navigation" occurs in both methods. Given that a unified model for the assessment will include the union of assessment models included in the methods eQual and Ahn, it should also include the criteria found in these models. Therefore, ancestors of the criterion of "availability" should be characteristics included in the Ahn and unified model. The criterion "ease_of learn_to_operate" should be in the form of ancestral characteristics in eQual model and the unified model. In contrast, the ancestors of the criterion "ease_of_navigation" should be the characteristics of each of the three models. However, such a link between criteria with multiple characteristics using subsumption relations should not occur, as this would mean redundancy. A redundant

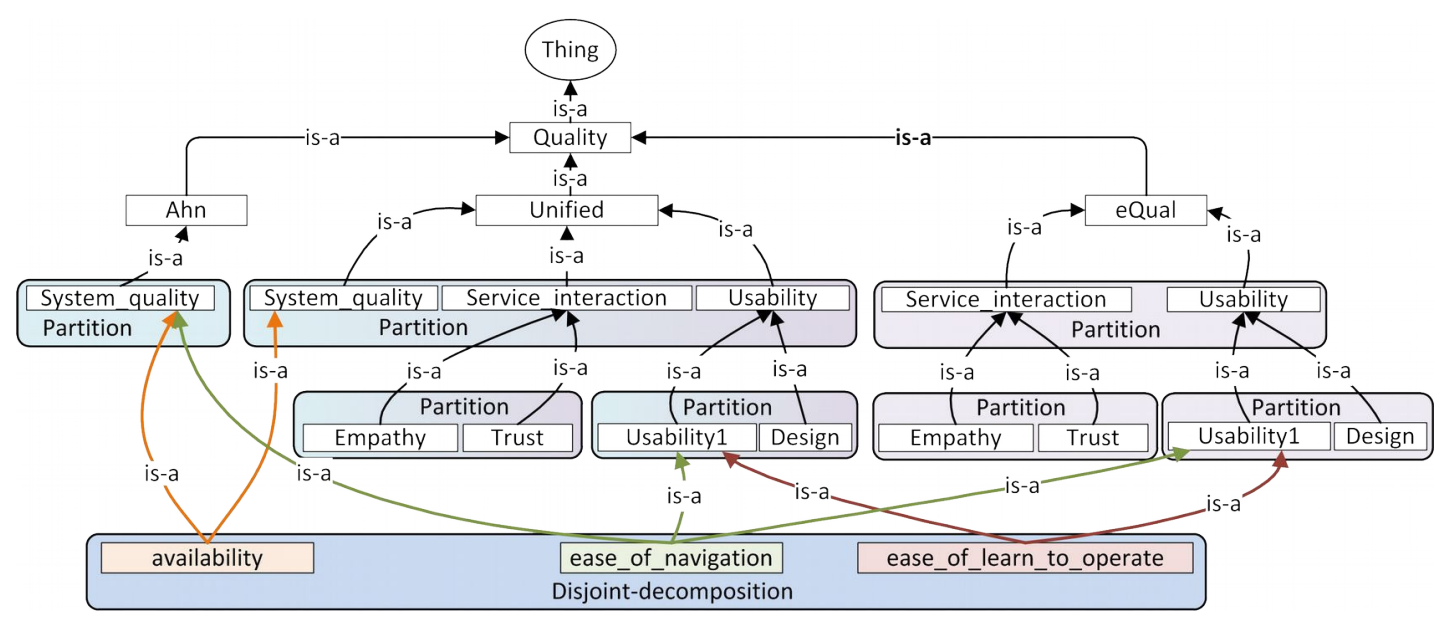

Fig 3. The problem of criteria membership for the evaluation models included in the target ontology 
occurrence of a subsumption relationship is in turn treated as an error in the method of integration formulated in [35]-[38].

The problem presented above can be solved through the method of use adopted by the authors of the ontology. Namely, at the stage of the source ontology specification, it was found that the constructed ontology criteria would be separated from the characteristics. This approach allowed for applications with greater transparency of the ontology. This is also consistent with the representation of open and closed world assumptions in the ontology [39]. Namely, the each quality model contained in the various assessment models is a closed model. This means that it is complete, and a new one cannot be added to it. The quality evaluation criteria are the open portion of the world, which means that there may be additional criteria that are not yet included in the ontology. For these reasons, the part of ontology reflecting the quality model was used to partition taxonomic relationships between concepts occurring at the same level of hierarchy. In contrast, disjoint-decomposition taxonomic relationship was among the criteria used, as these criteria describe the different parts' quality, but there may be other criteria for describing the quality. This solution allows for independent criteria of quality models in certain ways. In addition, it allows for inclusion in the ontology of many quality models, including the unified model. Therefore, the hierarchy of concepts of a target ontology should be similar to structure shown in Figure 4, which is the result of the integration of ontologies eQual and Ahn. Figure 4 shows a hypothetical ontology with three quality models eQual, Ahn and model obtained as a result of their unification. Furthermore, in the illustrated ontology, regardless of the quality models the criteria belonging to these models were incporporated.

To get a similar structure as that shown in Figure 4, a compromise between a fully integrated ontology and so called bridge ontology was chosen [34]. Namely, the target ontology should include a fully unified model of quality, including the characteristics derived from the source ontologies, along with the methods of assessment, for which the source ontologies were built. Additional models, such as eQual and Ahn, as well as quality characteristics, should be included in the target ontology in the form of components, which can be simply described as external. This will avoid any inconsistencies, where the same quality characteristics will occur in the different models. An example may be the characteristics of "Information_quality" appearing in the eQual and Ahn models. As a result of the full integration, only one such characteristic appears in the resulting ontology, and it would be included in the unified model and the eQual and Ahn models. This would include criteria that belonged to both eQual and Ahn model. Consequently, in each of these models, union of information quality criteria has existed. These models would not be as consistent with their actual structure, as defined in the relevant methods of quality assessment. The solution of this problem, which allows for the incorporation of quality models (eg. eQual and Ahn) as "external", but simultaneously makes it possible to include them in the target ontology, is go find a use for them other than for IRI identifiers' unified ontology.

Separating the criteria of the quality characteristics of ontology concept hierarchies' source requires that the reasoner infers membership criteria for the relevant characteristics of the subsumption relation linking them. For this purpose, the criteria should be linked to the characteristics using the appropriate relationship, as shown in Figure 5.

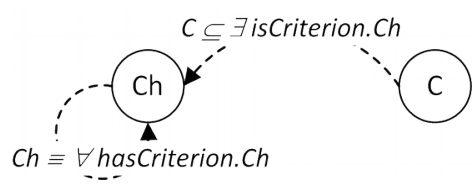

Fig 5. Proper configuration of relation "isCriterion" and "hasCriterion"

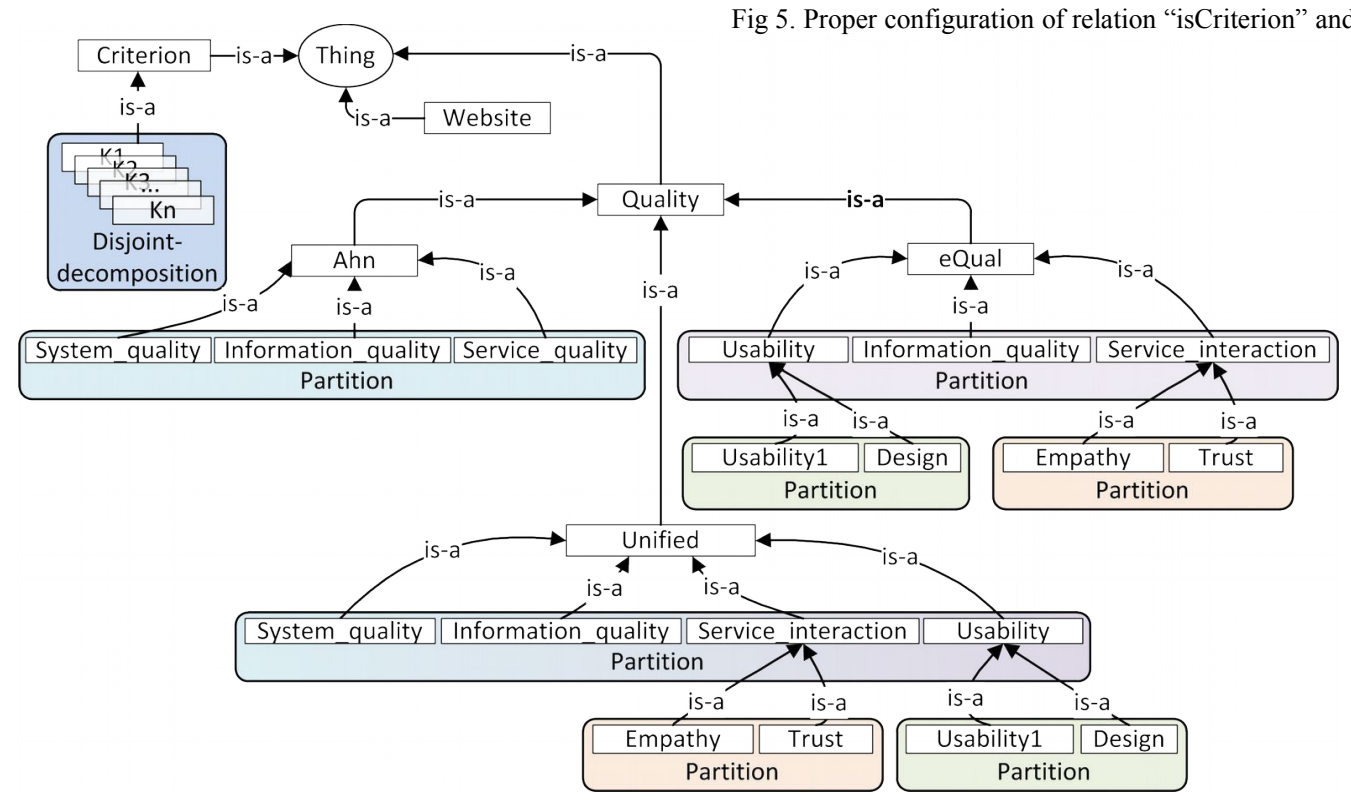

Fig 4. Hypothetical effect of eQual and Ahn integration in the target ontology 
The diagram shown in Figure 5 contains concepts $\mathrm{Ch}$ and $\mathrm{C}$, where $\mathrm{Ch}$ represents any quality characteristics, and $\mathrm{C}$ defines any criterion. By analyzing Figure 5 , it can be seen that the relation "hasCriterion" with a universal quantifier is established as a necessary and sufficient condition ( $\equiv)$ Ch concept, which is also the domain and scope of this relationship. The relationship "isCriterion" of the existential quantifier is a necessary condition $(\subseteq)$ criterion $\mathrm{C}$, for which coverage is a quality characteristic $\mathrm{Ch}$. This solution can be understood as follows: (a) there are certain criteria in the $\mathrm{C}$ group that belong to the characteristics of the Ch group ("C isCriterion some $\left.\mathrm{Ch}^{\prime}\right)$, (b) the characteristics of $\mathrm{Ch}$ are only those criteria that belong to the characteristic ("Ch hasCriterion only $\mathrm{Ch}^{\prime \prime}$ ). This configuration relationship between "isCriterion" and "hasCriterion" allows for the exploration of membership criteria to the characteristics and describe this kind of membership as a comprising specific criteria in the relevant characteristics. Presented in the form indicated, the relationship is very important, as our study showed that a different configuration could lead to erroneous conclusions inferred by the reasoner. An example of such a configuration error in the indicated relationships is included in Figure 6.

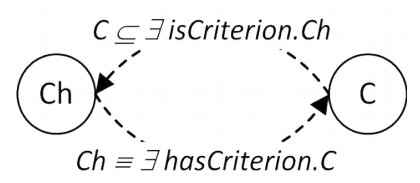

Fig 6. Wrong configuration of relation "isCriterion" and "hasCriterion"

Figure 6 shows a configuration in which the relationship "has Criterion" is marked as a necessary and sufficient condition $(\equiv)$ with an existential quantifier, and the scope of this relationship is criterion $\mathrm{C}$. This formulation relationship "hasCriterion" could cause inconsistent ontology at the level of the relationship. This will be explained by the example provided in Figure 7. In addition, will be used to clarify the description of the reasoning process, saved using descriptive logic expressions (1) - (12).

$$
\begin{gathered}
\text { Usability } \equiv \text { Design } \cup \text { Usability } 1 \\
\text { Design } \equiv \neg \text { Usability1 }
\end{gathered}
$$

Design $\equiv \exists$ hasCriterion.appropriateness_design

Usability $1 \equiv \exists$ hasCriterion.ease of navigation

System_quality $\equiv \exists$ hasCriterion.ease_of_navigation

System_quality $\equiv \exists$ hasCriterion.appropriateness_design_style

appropriateness_design $\equiv$ appropriateness_design_style

from (4), (5) Usability1 $\equiv$ System_quality

from (7) $\quad \exists$ hasCriterion.appropriateness_design ...

from (9),(3),(6) Design $\equiv$ System_quality

from(8),(10) Design $\equiv$ System_quality $\equiv$ Usability 1

from $(2),(11) \quad($ Design $\equiv$ Usability 1$) \wedge($ Design $\equiv \neg$ Usability1 $) \equiv \varnothing$

Figure 7 shows a portion of the target ontology, which is a hypothetical result of the integration of eQual and Ahn. The contents of Figure 7 include the quality characteristics eQual, i.e. "Design" and "Usability1", which make up the partition (i.e. they are disjoint and fully fill the space of eQual concept), which describes the axioms (1) and (2). In addition, Figure 7 contains the concepts of "ease_of_navigation", "appropriateness_design" and "appropriateness_design_style", which are some of the criteria. The criterion of "ease of navigation" is used in the Ahn method in the characteristics of "System_quality" and the method eQual characteristics "Usability1". The criterion of "appropriateness_design" occurs in the characteristics of the "Design" eQual method and an

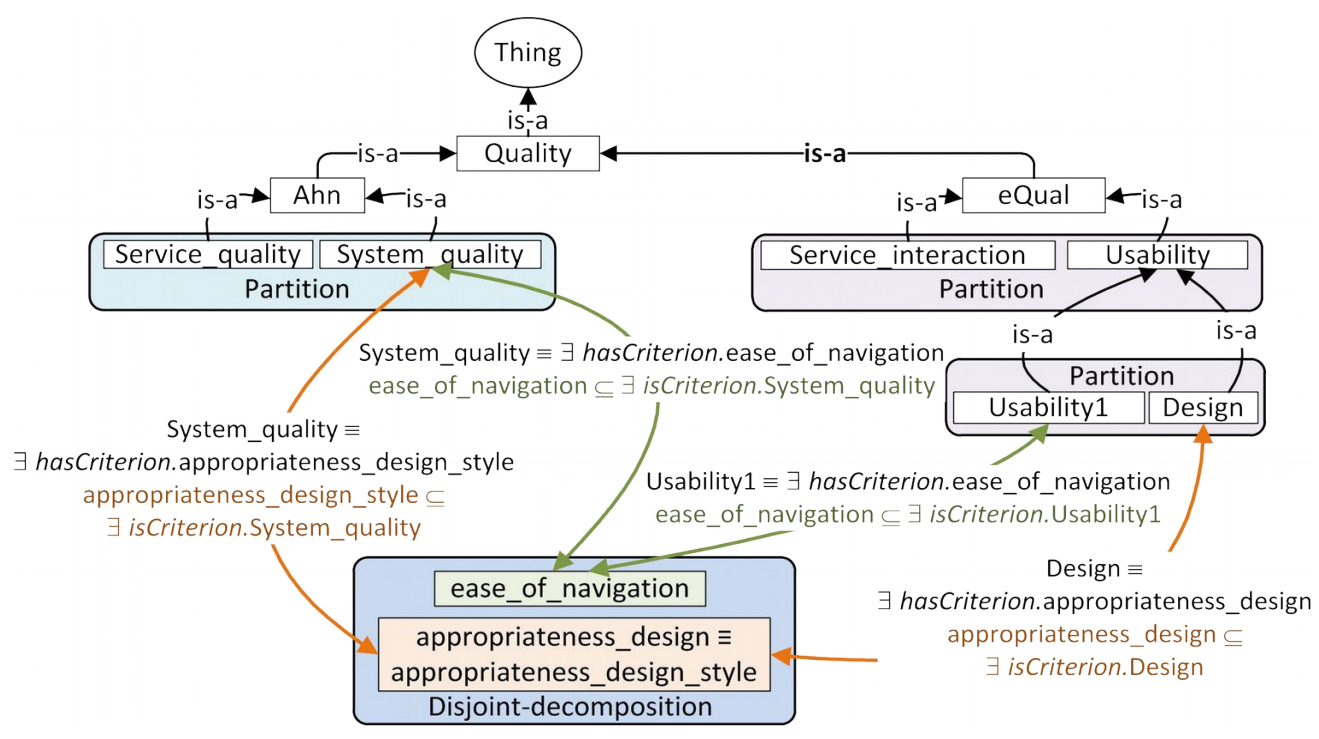

Fig 7. Relationships between criterion and characteristics which raise the inconsistency inference 
"appropriateness_design_style" functions in the characteristics of "System_quality" Ahn method. In addition, the concepts of "appropriateness_design" and "appropriateness_design_style" were considered to be equivalent to (7). Therefore, the concepts relating to design in an integrated ontology will be linked to the relationship "isCriterion" both with the concept of "System_quality" as well as "Design". The concept of "ease_of_navigation" in an integrated ontology will be linked to the relationship "isCriterion", the concepts "Usability1" and "System_quality". Assuming that:

- the concepts "Design" and "System_quality" are necessary and sufficient conditions include relations "hasCriterion some appropriateness_design" and "hasCriterion some appropriateness_design_style" (expressions (3) and (6)),

- then the concepts "Usability1" and "System_quality", as necessary and sufficient conditions, would cover the relationship "hasCriterion some ease_of_navigation" (expressions (4) and (5)),

during the integration of ontology, inconsistency would arise if the reasoner effect and "hasCriterion" relationships are defined as the necessary and sufficient conditions. If, for example the concept of "System_quality" has the necessary and sufficient condition "hasCriterion some ease_of_navigation", then it shall be deemed to be equivalent to the reasoner, the other concepts having the same condition. Therefore, it will be deemed to be equivalent to the concept of "Usability1" (8). On the other hand, based on a necessary and sufficient condition, "hasCriterion some appropriateness_design_style" shall be deemed equivalent to the concept of "Design", which has an equivalent condition "hasCriterion some appropriateness_design" (9), (10). The situation will appear when the concept of "System_quality" is equivalent to the concepts "Usability1" and "Design" (11). Accordingly, the reasoner also recognizes that these concepts are equivalent (12). The fact that the concepts of "Usability1" and "Design" create a partition is important, so that they are mutually exclusive and can not be considered equivalent to (12). This raises the inconsistency inference. The example given there is that there is no inconsistency in the target ontology due to the fact that the relationship "hasCriterion" and "isCriterion" between concepts of characteristics and quality criteria are defined as shown in Figure 5.

During integration there may be other inconsistencies that must be addressed on a regular basis. This will be discussed using the example of concepts called "Criterion" coming from the ontology eQual and SiteQual. In view of the fact that the method eQual grading scale covers a range of values from 1 to 7, a restriction should be imposed on the concept of "Criterion" in the ontology eQual " hasEvaluationValue only integer $[>=1,<=7]$ ". Meanwhile, in the SiteQual method the evaluation of criteria includes the values from 1 to 9, so that the concept of "Criterion" in the ontology describing this method will be limited to "hasEvaluationValue only integer $[>=1,<=9]$ ". While the integration of ontologies these concepts should be unified, the question arises: "What range of values should include restricting the concept of "Criterion" in a unified model of the target ontology?". In order to solve the conflicts mentioned, we decided to adopt a solution from the PROMPT algorithm. It occurs when one of ontology is considered to be the preferred one and conflicts of this type are resolved in its favor [40]. During the integration, target ontology that unifying source ontologies will be the preferred. Therefore, the order of the integrated source ontology will have a partial effect on the resulting form, a unified model of the target ontology.

Based on these observations, conclusions and guidelines, the source ontologies was built, reflecting the website evaluation quality methods. These ontologies, in the form of primary evidence, and inferred by the reasoner, are presented in: eQual [41], Ahn [42] SiteQual [43] Website Evaluation Questionnaire [44] and Web Portal Site Quality [45]. More of the construction process of source ontologies is described in [46]. Based on the literature analysis presented earlier, feedback and observations, we formulated the integration algorithm that was then applied in the process of ontology integration of website quality evaluation methods.

\section{SOURCE ONTOlOGIES INTEGRATION ALGORITHM IN THE TARGET ONTOLOGY}

The developed ontology integration algorithm is closely related to refactoring and ontology merging support tools offered by Protégé editor [47]. These tools make it much easier to carry out the integration process. The integration algorithm is divided into three parts. Part I is performed only once as part of the integration process. It concerns the preparation for the integration and the integration of the first source ontology in the target ontology. Part II includes the integration of the criteria and the placement in the target ontology the individual models of evaluation coming from the integrated ontology, as with the methods that are reflected by the different source ontologies. Part III deals with the construction of a unified quality model in the target ontology.

Part I: Preparing for the integration and integration of the first ontology. As a result of the first part of the algorithm, contained in the target ontology is the first source ontology. This part consists of four steps.

1. In order to prepare for integration, new empty ontology should be created in the Protégé editor, which are then integrated source ontologies. The created ontology IRI identifier must be given a value of "Integrated".

2. Then the first source ontology for integration should be selected; open the target ontology "Integrated" in editor Protégé and import the selected source ontology (e.g. "eQual"). 
3. The next step is to use the "Merge ontology" option in order to merge the source ontology (e.g. "eQual") and the destination ("Integrated"). In this way, the two ontologies will be included in the same file ontology.

4. Then, using refactoring tools, change the IRI identifier of the source ontology elements to "Integrated" value, which is the IRI identifier of the target ontology. This should be performed for all elements of the source ontology, with the exception of the concepts contained therein describing the quality model (for "eQual" the following concepts should be left unchanged: "eQual", "Service_interaction", "Empathy", "Trust", "Information_quality", "Usability", "Design" and "Usability1"). This action will preserve the quality model of the source ontology in the target ontology.

Part II: Integration of another source ontology in the section containing the criteria and quality model. As a result of this part of the algorithm, another source ontology will be placed into the target ontology (another website evaluation quality model and their criteria). It consists of two stages, having in total six steps.

Stage I: Selection of another source ontology to integration and alignment of parts of the source and target ontologies containing criteria.

1. Next source ontology for integration should be selected.

2. Then, to align the parts of the ontologies containing criteria, identify the relationship between the quality criteria present in the target ontology (i.e. "Integrated" in accordance with its current content) and in the source ontology to be integrated into the next stage. To do this, use the dictionaries, thesaurus, toolsof the Protégé editor and source literature concerning approaches represented by ontologies.

Stage II: Integration of another source ontology.
1. With Protégé editor open the target ontology "Integrated", perform another source ontology imports (e.g. "Ahn").

2. Using the tool "Merge ontologies", connect the source ontology (e.g. "Ahn") and the target (e.g. "Integrated", which already contains concepts from "eQual").

3. The next step is to change the IRI of the previously assigned values (e.g. "Ahn") to "Integrated" for all elements from the source ontology except those that describe the quality model (for Ahn they are concepts, "Ahn", "Information_quality", "System_quality" and "Service_quality"). This action will fit together just called concepts of criteria of source and target ontologies. The relations and concepts that are rooted in the ontology, i.e. "Criterion", "Quality" and "Service", will also be matched in this way.

4. The next action is to introduce the relationships of equivalence and subsumption between concepts of criteria derived from the source and the target ontologies. These relationships should be identified at the stage of aligning criteria (Part II, Phase I, Step 2).

Part III: Building a unified model of quality. This part consists of three stages, having in total 4 steps that should be done at the end of Part II of the algorithm.

Stage I: Aligning quality models. You need to specify the link between quality characteristics included in the unified model. As in the alignment step occurring in the second part of the integration algorithm, a number of tools must also be used here to facilitate the identification of links.

Stage II: Construction of a unified model of quality. This model should include the characteristics contained in the source and target ontology. After determining its structure, the previous unified model contained in the target ontology should be replaced. Quality characteristics of a unified model should contain relationship "hasCriterion". The criteria should be related to the characteristics of the model relationship "isCriterion".

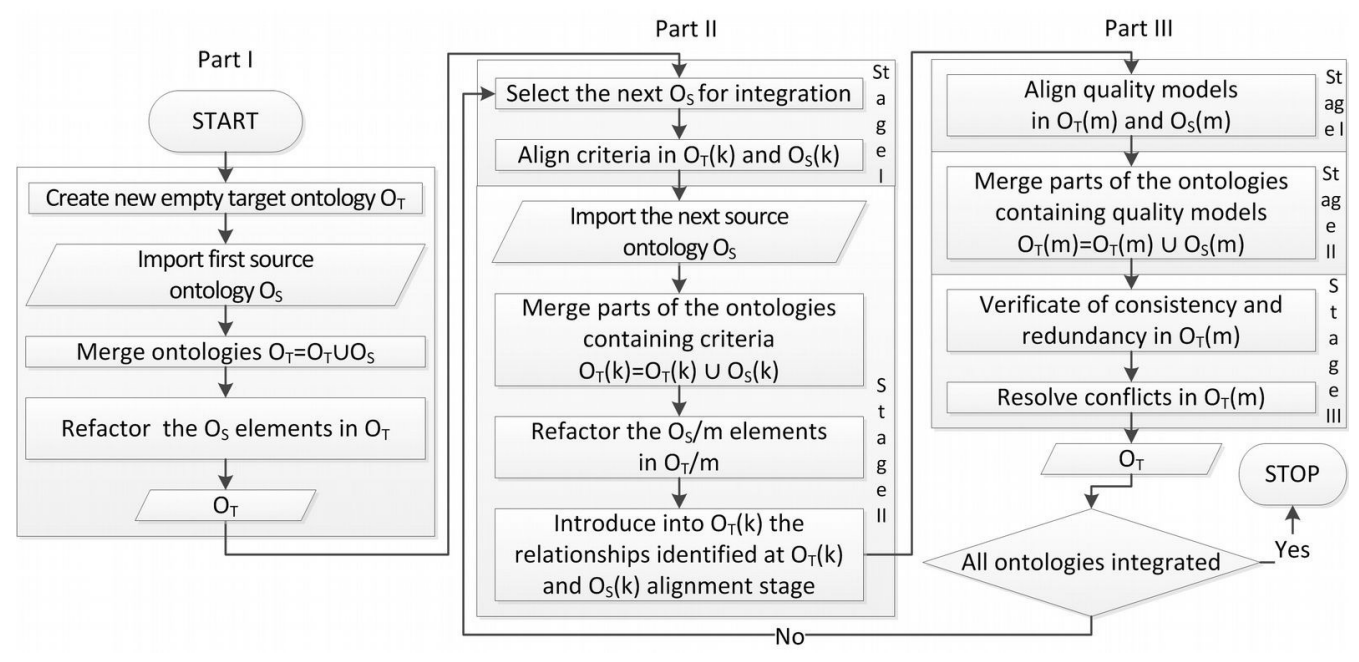

Fig 8. Algorithm for ontology integration 
Stage III: Verification of consistency and absence of redundancy and resolving conflicts.

1. This should focus on the consistency of the target ontology obtained using reasoner.

2. Conflicts involving two equivalent characteristics having different names can also occur here. Then you need to decide what the characteristics that will be included in the model should be called. Furthermore, one criterion may be associated by the relationship "isCriterion" with two characteristics included in the unified model. Such conflicts must be resolved by selecting the preferred characteristics and relationships derived from a previous version of the unified model.

A block diagram of the integration algorithm is shown in Figure 8 .

\section{RESUlts}

In order to integrate the source ontology built earlier, according to the algorithm presented in point IV, the first part of the process of integration was completed, i.e. the creation of an empty target ontology, and integrated in there the eQual source ontology. Then a second ontology was selected for integration, which was Ahn ontology, and the alignment of concepts representing criteria was carried out. In this step links were identified between the target ontology, which already contains eQual, and the source ontology Ahn. The links detected are shown in Table 1. It should be noted that the alignment of the ontologies created some problems. Namely, certain relationships between concepts may not be obvious. Therefore, in order to determine the relationships between concepts of criteria, dictionaries, thesauruses and source literature concerning methods of integration represented by ontologies were used. The second problem results from the the fact that the integrated ontologies are creations of secondary structures, developed on the basis of quality assessment methods. The criteria contained in the individual methods are typically in the form of sentences, so that their names are in the long form. Therefore, in the analysis of the links there may appear to be some confusion regarding the full wording of the concept.

Based on the identified relationship between the criteria set out in Table 1, the next stage of integration was carried out, i.e. a merging ontologies in Protégé editor. In this framework the following things were achieved: import Ahn source ontology to target ontology, ontology merging with the use of the tool "Merge ontologies", the change values of IRI identifiers for concepts / criteria in Ahn ontology, linking respective pairs of concepts with equivalent relationships. Therefore, the target ontology, which is a unified ontology eQual and Ahn at the level of criteria, was achieved.

Then the concepts representing the characteristics of quality models Ahn and eQual were aligned, as shown in Table 2. The relationship between the characteristics set out in Table 2 is presented in that way due to the fact that the criteria included in the particular characteristics were symlinked in it at the same level of hierarchy. For example, if the "Service quality" has been recognized as being equivalent to the characteristics of "Service_interaction", then e.g. the criterion of "instills_confidence " (belonging to the "Service_quality") would occur on the 3rd level of the proposed hierarchy along with the characteristics of the "Trust" (including the "Service_interaction"). The criterion of "reputation" (belonging to the "Trust") would be at level 4 , resulting thus in a hierarchy in which on one level in the context of the characteristics of "Service interaction" are both criteria and subcharacteristics. This could cause problems with inconsistency during the inference by the reasoner, so in this case these characteristics associated with subsumption relationship.

The last step was to check the consistency and lack of redundancy in an integrated ontology. Such redundancy appeared as a result of the reasoner, in the case of the criteria from the target and source ontology having the same name or having been recognized as equivalent, and belonging to two different characteristics included in the unified model. In each of these cases, there was a redundancy of subsumption relationship between the criteria and characteristics of the

TABle I. Aligning THE CONCEPTS OF CRITERIA IN THE TARGET ONTOLOGY CONTAINING THE EQUAL MODEL AND SOURCE ONTOLOGY AHN

\begin{tabular}{|c|c|c|c|c|c|}
\hline \multirow{2}{*}{ No. } & \multirow{2}{*}{ Relation type } & \multicolumn{2}{|c|}{ Target ontology (containing eQual) } & \multicolumn{2}{|c|}{ Source ontology Ahn } \\
\hline & & Characteristic & Criterion / Concept & Characteristic & Criterion / Concept \\
\hline 1. & equivalence & Usability1 & ease of navigation & System quality & ease of navigation \\
\hline 3. & equivalence & Design & sense of competency & Service quality & professionalism and competence \\
\hline 4. & equivalence & Design & positive experience & System quality & audio-visual experience \\
\hline 5. & equivalence & Information quality & information accuracy & Information quality & information accuracy \\
\hline 6. & equivalence & Information quality & information believability & Information quality & information reliability \\
\hline 7. & equivalence & Information quality & information timeliness & Information quality & information timeliness \\
\hline 8. & equivalence & Information quality & appropriate format of information & Information quality & appropriate format of information \\
\hline 9. & equivalence & Trust & security of personal information & System quality & security of personal information \\
\hline 10. & equivalence & Empathy & personalization & Service quality & adaptation to the user's needs \\
\hline 11. & equivalence & Trust & confident about delivery goods and services & Service quality & providing whatever promised \\
\hline
\end{tabular}


TABLE II. ALIGNING THE CONCEPTS OF QUALITY MODEL IN THE TARGET ONTOLOGY CONTAINING THE EQUAL MODEL AND SOURCE ONTOLOGY AHN

\begin{tabular}{|c|c|c|c|c|c|}
\hline \multirow{2}{*}{ No. } & \multirow{2}{*}{ Relation type } & \multicolumn{2}{|c|}{ Target ontology (containing eQual) } & \multicolumn{2}{c|}{ Source ontology Ahn } \\
\cline { 3 - 6 } & & Parent concept & Characteristic & Parent concept & Characteristic \\
\hline 1. & equivalence & Quality & Information quality & Quality & Information quality \\
\hline 2. & reverse subsumption & Quality & Service interaction & Quality & Service quality \\
\hline
\end{tabular}

unified model. Selected examples of such redundancy are presented in Figure 9, which shows both the redundant relationship within one sheet, as well as within different characteristics.

For example, a couple of equivalent concepts "personalisation" and "adaptation_to_the_user's_needs" belongs to the characteristics of the "Service_quality" and "Empathy". On the other hand, the subcharacteristics are included in the single characteristic "Service_interaction". So here there is a redundancy in a single sheet. This redundancy resulted from the fact that the criterion of "personalisation" in the ontology eQual being part of the category of "Empathy" and the criterion of "adaptation_to_the_user's_needs" in Ahn ontology being one of the characteristics of "Service_quality". As a result of the recognition of these criteria as being equal to each other, they take the relationship between them and the characteristics to which they belong. A similar situation occurs, for example, in the case of matched pairs of concepts "sense_of_competency" and "professionalism_and competence", which are among the characteristics of "Service_quality" and "Design" as a result of the equivalence that exists between them. This type of redundancy relationship is resolved in accordance with the assumptions described in Section III of the article, i.e. for the benefit of the target ontology, keeping derived from the relationship, and removing accounts from the source ontology (in this case from the ontology Ahn). Integrating ontology models eQual and Ahn and including the quality model unifies these two methods, presented in the elementary form [48], in the form deduced by the reasoner [49].
Other iterations of the process of integration of the various source ontology and of the target ontology integration proceeded analogously to those presented above. Previously built ontologies accompanied the target ontology in turn systematically: SiteQual, Website Evaluation Questionnaire and Web Portal Site Quality [50]. Therefore, the ontology [50] contains five source ontologies. For the unified model of quality, consisting of five basic models as deduced by the reasoner, each model is presented separately in [51].

\section{Conclusion}

Even a cursory analysis of [50] and [51] shows a very high level of complexity in this built ontology. At the same time, it can be concluded that the construction of such an extensive ontology, as a result of the integration of the source ontologies, is much less complex than its construction process from scratch, even with the use of formal ontology construction methodology. Thanks to the presented approach, the problem of ontology construction unifying quality assessment methods was decomposed for a few minor problems involving construction of the source ontologies were integrated. This resulted in an ontology containing quality models used in the various methods and a model that unifies the different methods. It is worth noting that in all of the integrated methods a total of 115 criteria were used. In the integrated ontology, 94 criteria are listed, due to the fact that part of the criteria are repeated in the various methods. However, when one takes into account the fact that some criteria are equivalent to those of others, one can consider there to be 70 different quality criteria in an integrated ontology. Therefore, we managed to limit the number of applied ontology criteria by almost $40 \%$. The resulting

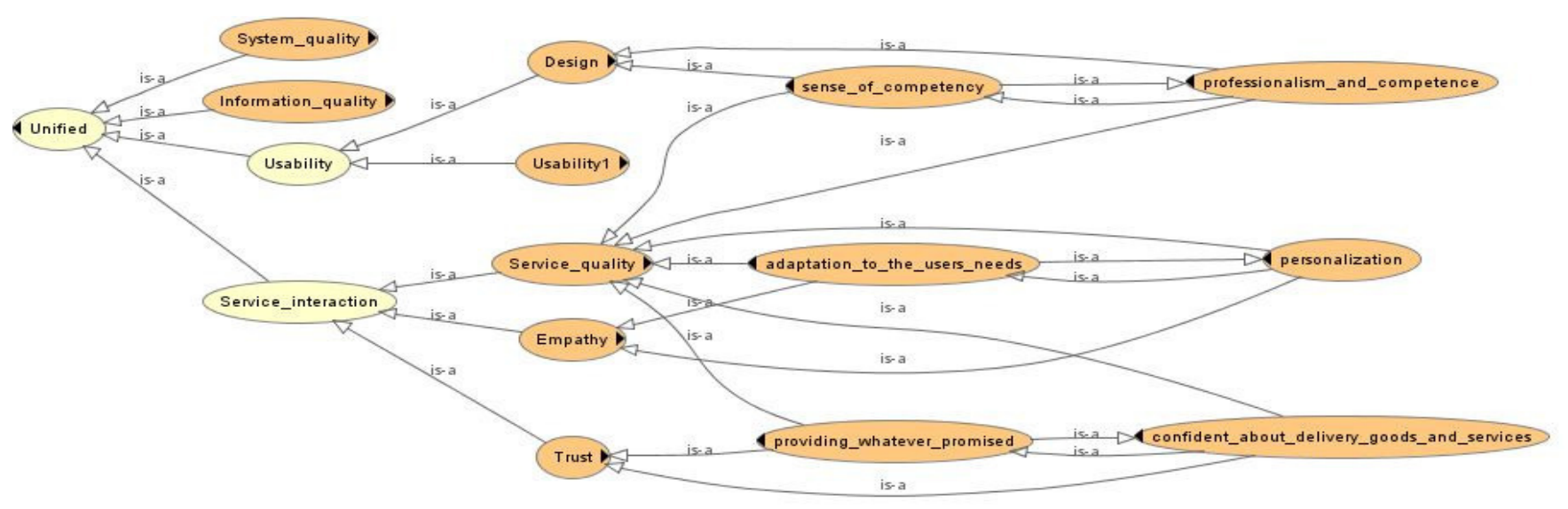

Fig 9. Redundancy of relationships in the target ontology containing the source ontologies eQual and Ahn 
ontology presented in [51] can act as a repository of domain knowledge, due to the fact that it includes a number of methods and models that evaluate the quality of websites. It may also allow for the integration of heterogeneous data from a variety of assessment methods, and thus assessment websites through a variety of methods defined in the ontology, so that the individual results of the assessment can be compared in a the same terminology and a reference plane. Constructed repository of knowledge, together with presented in [16] website assessment criteria selection process could be at the core of the expert system of website quality assessment, which should be the direction of further research. In addition, further work should include the development of ontology about the possibility of environmental data records about ontology users.

\section{ACKNOWLEDGMENT}

The work was partially supported by Fellowship coFinanced by European Union within European Social Fund, by European Union's Seventh Framework Programme for research, technological development and demonstration under grant agreement no 316097 [ENGINE].

\section{REFERENCES}

[1] W.C. Chiou, C.C. Lin, C. Perng, "A strategic framework for website evaluation based on a review of the literature from 1995-2006," Information \& Management, vol. 47, no. 5-6, pp. 282-290, 2010, http://dx.doi.org/10.1016/j.im.2010.06.002

[2] IAB, IAB internet advertising revenue report. 2013 full year results. PricewaterhouseCoopers LLP, 2014.

[3] IAB Europe, Adex Benchmark 2013. European online advertising expenditure.IAB Europe, 2014

[4] ECOMMERCE Europe, European B2C E-commerce Report 2014 www.ecommerce-europe.eu, 2014.

[5] S. Kim, L. Stoel, "Dimensional hierarchy of retail website quality," Information \& Management, vol. 41, no. 5, pp. 619-633, 2004.

[6] J. Hwang, Y.S. Yoon, N.H. Park, "Structural effects of cognitive and affective reponses to web advertisements, website and brand attitudes, and purchase intentions: The case of casual-dining restaurants,' International Journal of Hospitality Management, vol. 30, no. 4, pp. 897-907, 2011, http://dx.doi.org/10.1016/j.ijhm.2011.01.011

[7] W.C. Chou, Y. Cheng, "A hybrid fuzzy MCDM approach for evaluating website quality of professional accounting firms," Expert Systems with Applications, vol. 39, no. 3, pp. 2783-2793, 2012, http://dx.doi.org/10.1016/j.eswa.2011.08.138

[8] ISO/IEC 25010:2010(E), Systems and software engineering Systems and software Quality Requirements and Evaluation (SQuaRE) - System and software quality models.

[9] S.J. Barnes, R. Vidgen, "Data triangulation and web quality metrics: A case study in e-government," Information \& Management, vol. 43, no. 6 , pp. 767-777, 2006.

[10] T. Ahn, S. Ryu, I. Han, "The impact of Web quality and playfulness on user acceptance of online retailing," Information \& Management, vol. 44 , no. 3 , pp. $263-275,2007$

[11] H.W. Webb, L.A. Webb, "SiteQual: an integrated measure of Web site quality," Journal of Enterprise Information Management, vol. 17 no. 6, pp. 430-440, 2004.

[12] Z. Yang, S. Cai, Z. Zhou, N. Zhou, "Development and validation of an instrument to measure user perceived service quality of information presenting Web Portals," Information \& Management, vol. 42 , no. 4 , pp. 575-589, 2005

[13] S. Elling, L. Lentz, M. de Jong, H. van den Bergh, "Measuring the quality of governmental websites in a controlled versus an online setting with the 'Website Evaluation Questionnaire'," Government Information Quarterly, vol. 29, no. 3, pp. 383-393, 2012.

[14] H. Sorum, K.N. Andersen, T. Clemmensen, "Website quality in government: Exploring the webmaster's perception and explanation of website quality," Transforming Government: People, Process and Policy, vol. 7, no. 3, pp. 322-341, 2013, http://dx.doi.org/10.1108/TG-10-2012-0012

[15] T. Kaya, "Multi-attribute Evaluation of Website Quality in E-business Using an Integrated Fuzzy AHPTOPSIS Methodology," International Journal of Computational Intelligence Systems, vol. 3, no. 3, pp. 301314, 2010, http://dx.doi.org/10.1080/18756891.2010.9727701

[16] P. Ziemba, M. Piwowarski, J. Jankowski, J. Wątróbski, "Method of Criteria Selection and Weights Calculation in the Process of Web Projects Evaluation," Lecture Notes in Artificial Intelligence, vol. 8733, pp. 684-693, 2014, http://dx.doi.org/10.1007/978-3-31911289-3_69

[17] M. Hepp, "Ontologies: state of the art, business potential, and grand challenges," in Ontology Management. Semantic Web, Semantic Web Services, and Business Applications, M. Hepp, P. de Leenheer, A. de Moor, Y. Sure, Ed. Heidenberg: Springer, 2008, pp. 3-23.

[18] I.F. Cruz, H. Xiao, "Ontology Driven Data Integration in Heterogeneous Networks," Studies in Computational Intelligence, vol. 168 , pp. 75-98, 2009.

[19] T.R. Gruber, "A translation approach to portable ontology specifications," Knowledge Acquisition, vol. 5, no. 2, pp. 199-220, 1993.

[20] A. Guzman-Arenas, A.D. Cuevas, "Knowledge accumulation through automatic merging of ontologies," Expert Systems with Applications, vol. 37, no. 3, pp. 1991-2005, 2010, http://dx.doi.org/10.1016/j.eswa.2009.06.078

[21] N. Villanueva-Rosales, M. Dumontier, "yOWL: An ontology-driven knowledge base for yeast biologists," Journal of Biomedical Informatics, vol. 41, no. 5, pp. 779-789, 2008.

[22] L.Y. Shue, C.W. Chen, W. Shiue, "The development of an ontologybased expert system for corporate financial rating," Expert Systems with Applications, vol. 36, no. 2, pp. 2130-2142, 2009, http://dx.doi.org/10.1016/j.eswa.2007.12.044

[23] R. Saa, A. Garcia, C. Gomez, J. Carretero, F. Garcia-Carballeira, “An ontology-driven decision support system for high-performance and cost-optimized design of complex railway portal frames," Expert Systems with Applications, vol. 39, no. 10, pp. 8784-8792, 2012, http://dx.doi.org/10.1016/j.eswa.2012.02.002

[24] B. Magoutas, C. Halaris, G. Mentzas, "An Ontology for the Multiperspective Evaluation of Quality in E-Government Services," Lecture Notes in Computer Science, vol. 4656, pp. 318-329, 2007.

[25] L. Mich, M. Franch, "Instantiating Web Sites Quality Models: an Ontologies driven Approach," in Proceedings of the CAISE'05 Workshop on Web Oriented Software Technologies, 2005.

[26] N. Casellas, Legal Ontology Engineering. Methodologies, Modelling Trends, and the Ontology of Professional Judicial Knowledge. Dordrecht: Springer, 2011, http://dx.doi.org/10.1007/978-94-0071497-7

[27] O. Corcho, M. Fernandez-Lopez, A. Gomez-Perez, "Ontological Engineering: Principles, Methods, Tools and Languages," in Ontologies for Software Engineering and Software Technology, C. Calero, F. Ruiz, M. Piattini, Ed. Berlin: Springer, 2006, pp. 1-48.

[28] Y. Sure, C. Tempich, D. Vrandecic, "Ontology Engineering Methodologies. in Semantic Web Technologies. Trends and Research," in Ontology-based Systems, J. Davies, R. Studer, P. Warren, Ed. Chichester: Wiley, 2006, pp. 171-190.

[29] M. Fernandez-Lopez, A. Gomez-Perez, "Overview and analysis of methodologies for building ontologies," The Knowledge Engineering Review, vol. 17, no. 2, pp. 129-156, 2002

[30] A. Gomez-Perez, M. Fernandez-Lopez, O. Corcho, "Methodologies and Methods for Building Ontologies," in Ontological Engineering, With Examples from the Areas of Knowledge Management, eCommerce and the Semantic Web, A. Gomez-Perez, M. FernandezLopez, O. Corcho, Ed. London: Springer, 2004, pp. 107-197.

[31] O. Corcho, M. Fernandez-Lopez, A. Gomez-Perez, A. Lopez-Cima, "Building Legal Ontologies with METHONTOLOGY and WebODE," Lecture Notes in Computer Science, vol. 3369, pp. 142-157, 2005. 
[32] B. Villazon-Terrazas, J. Ramirez, M.C. Suarez-Figueroa, A. GomezPerez, "A network of ontology networks for building e-employment advanced systems," Expert Systems with Applications, vol. 38, no. 11, pp. 13612-13624, 2011, http://dx.doi.org/10.1016/j.eswa.2011.04.125

[33] H. Pinto, A. Gomez-Perez, J. Martins, "Some issues on ontology integration," in Proceedings of IJCAI99's Workshop on Ontologies and Problem Solving Methods, 1999.

[34] J. de Bruijn, M. Ehrig, C. Feier, F. Martin-Recuerda, F. Scharffe, M. Weiten, "Ontology Mediation, Merging, and Aligning," in Semantic Web Technologies. Trends and Research in Ontology-based Systems, J. Davies, R. Studer, P. Warren, Ed. Chichester: Wiley, 2006, pp. 171-190.

[35] M. Klein, "Combining and relating ontologies: an analysis of problems and solutions," in Proceedings of the IJCAI Workshop on Ontologies and Information Sharing, 2001, pp. 53-62.

[36] J. Zhang, Y. Lv, "An Approach of Refining the Merged Ontology," in Proceedings of the 9th IEEE International Conference on Fuzzy Systems and Knowledge Discovery, 2012, pp. 802-806, http://dx.doi.org/10.1109/FSKD.2012.6233973

[37] M. Taboada, D. Martinez, J. Mira, "Experiences in reusing knowledge sources using Protege and PROMPT," International Journal HumanComputer Studies, vol. 62, no. 5, pp. 597-618, 2005.

[38] M. Gaeta, F. Orciuoli, P. Ritrovato, "Advanced ontology management system for personalised e-Learning," Knowledge-Based Systems, vol. $22, \quad$ no. $4, \quad$ pp. 2009 , http://dx.doi.org/10.1016/j.knosys.2009.01.006
[39] M. Knorr, J.J. Alferes, P. Hitzler, "Local closed world reasoning with description logics under the well-founded semantics," Artificial Intelligence, vol. 175, no. 9-10, pp. 1528-1554, 2011, http://dx.doi.org/10.1016/j.artint.2011.01.007

[40] N.F. Noy, M.A. Musen, "The PROMPT suite: interactive tools for ontology merging and mapping," International Journal of HumanComputer Studies, vol. 59, no. 6, pp. 983-1024, 2003.

[41] http://tinyurl.com/WQM-eQual-example-inst; http://tinyurl.com/WQM-eQual-inferred-example-ins

[42] http://tinyurl.com/WQM-Ahn; http://tinyurl.com/WQM-Ahn-inferred

[43] http://tinyurl.com/WQM-SiteQual; http://tinyurl.com/WQMSiteQual-inferred

[44] http://tinyurl.com/WQM-WEQ; http://tinyurl.com/WQM-WEQinferred

[45] http://tinyurl.com/WQM-WPSQ; http://tinyurl.com/WQM-WPSQinferred

[46] P. Ziemba, J. Jankowski, J. Wątróbski, J. Becker, "Knowledge Management in Website Quality Evaluation Domain," Lecture Notes in Artificial Intelligence, vol. 9330, pp. 75-85, 2015, http://dx.doi.org/10.1007/978-3-319-24306-1 8

[47] http://protege.stanford.edu/

[48] http://tinyurl.com/ont-integration1

[49] http://tinyurl.com/ont-integration1-inferred

[50] http://tinyurl.com/ont-integration

[51] http://tinyurl.com/ont-integration-inferred 